\title{
Polymorphic Ventricular Tachycardia with a Normal QTc Interval in a Patient with COVID-19 and Fever: Case Report
}

\author{
Savalan Babapoor-Farrokhran ${ }^{1}$ (D) $\cdot$ Zachary Port $^{1} \cdot$ Philip C. Wiener $^{1} \cdot$ Aman Amanullah $^{1,2} \cdot$ Sumeet K. Mainigi ${ }^{1,2}$
}

Accepted: 17 September 2020 / Published online: 24 September 2020

(C) Springer Nature Switzerland AG 2020

\begin{abstract}
Arrhythmias or conduction system disease are not the most common manifestation of COVID-19 infection in patients requiring hospital admission. Torsade de pointes typically occurs in bursts of self-limiting episodes with symptoms of dizziness and syncope. However, it may occasionally progress to ventricular fibrillation and sudden death. In this article, we report a case of COVID-19 patient who developed polymorphic ventricular tachycardia with torsade de pointes morphology with normal QTc interval in the setting of fever. An 81-year-old woman was admitted with symptoms of COVID-19. She was treated with hydroxychloroquine, azithromycin, and doxycycline at an outside facility and finished the treatment 5 days prior to admission to our facility. Her course was complicated by atrial fibrillation with rapid ventricular response requiring cardioversion. Later, she developed two episodes of polymorphic ventricular tachycardia with TdP morphology with normal QTc. There was a correlation with fever triggering the ventricular tachycardia. We advocated aggressive fever control given the QTc was normal and stable. Following fever control, the patient remained stable and had no abnormal rhythm. COVID-19 patients are prone to different arrhythmias including life-threatening ventricular arrhythmias with normal left ventricular systolic function and normal QTc, and they should be monitored for fever and electrolyte abnormality during their hospital stay.
\end{abstract}

Keywords Polymorphic ventricular tachycardia $\cdot$ Torsade de pointes · Arrhythmias · COVID-19 - Severe acute respiratory syndrome coronavirus- 2

\section{Introduction}

COVID-19 is considered to be a multi-organ system disease that can cause lung, liver, kidney, nervous system, and reproductive system damage [1]. Arrhythmias or conduction system disease are not the most common manifestation of COVID-19 infection in patients requiring hospital admission. Myocardial injury is common in critically ill COVID-19 individuals as a result of different plausible mechanisms including direct damage to the cardiomyocytes, systemic inflammation, interferon-mediated immune response, exaggerated cytokine

This article is part of the Topical Collection on Covid-19

Savalan Babapoor-Farrokhran

Babapoos@einstein.edu

1 Division of Cardiology, Department of Medicine, Einstein Medical Center, 5501 Old York Road, Philadelphia, PA 19141, USA

2 Sidney Kimmel Medical College, Thomas Jefferson University, Philadelphia, PA 19107, USA response by types 1 and 2 helper T cells, coronary plaque destabilization, and hypoxia $[2,3]$.

Ventricular arrhythmia may be either monomorphic with a single QRS morphology or polymorphic with QRS morphology varying in amplitude, axis, and duration. The most common cause of polymorphic ventricular tachycardia (PVT) is acute myocardial ischemia, which may quickly progress to ventricular fibrillation (VF). Less commonly, PVT is caused by circumstances that prolong the QT interval (drugs and ionchannel disorders) [4]. Other risk factors for PVT include baseline QT prolongation, bradycardia, and electrolyte disturbances (especially profound hypokalemia and hypomagnesemia, and less often hypocalcemia). Acquired long QT syndrome (LQTS) can occur due to a variety of factors including medications and electrolyte derangements. Torsade de pointes (TdP) is a PVT that occurs when there is delayed ventricular repolarization with associated triggered activity due to early after depolarizations. It typically occurs in bursts of selflimiting episodes with symptoms of dizziness and syncope. However, it may occasionally progress to VF and sudden death [5]. In this article, we report a case of COVID-19 patient 
who developed polymorphic ventricular tachycardia with TdP morphology with normal QTc $(441 \mathrm{~ms})$ interval in the setting of fever.

\section{Case Presentation}

An 81-year-old woman with no cardiac history and past medical history of rheumatoid arthritis, hypothyroidism, and diabetes mellitus type 2 who presented as a transfer from an outside facility following acute infection of SARS-CoV-2. At the prior institution, she was treated with hydroxychloroquine and azithromycin and finished the course of treatment 5 days prior to admission to our hospital. Her course was complicated by paroxysmal atrial fibrillation with rapid ventricular response treated by direct current cardioversion and $3 \mathrm{~g}$ of amiodarone loading dose followed by $200 \mathrm{mg}$ of oral amiodarone daily for maintenance of sinus rhythm. Baseline electrocardiograms (ECG) on admission and during the initial hospital course were noted as normal sinus rhythm with a normal QT/QTc interval (441 ms, 467 ms) (Fig. 1a, b). Following admission to our facility, she experienced worsening mental status, hypoxia, and fever. She was treated with empiric antimicrobials including micafungin $150 \mathrm{mg}$ intravenous daily for 2 weeks and meropenem $2000 \mathrm{mg}$ intravenous every $8 \mathrm{~h}$ for 5 days (not known to prolong QT interval) [6]. She was also receiving multivitamins, folic acid, and subcutaneous Lovenox for deep venous thrombosis prophylaxis. Despite empiric antibiotic and antifungal coverage, she possessed a persistent fever and leukocytosis of 12,000 counts/
mcL. She was re-tested for SARS-CoV-2 using real-time reverse transcriptase-polymerase chain reaction (RT-PCR; Abbott ID) testing from a nasopharyngeal sample and was positive. Subsequently, she became unresponsive and noted to have two episodes of polymorphic ventricular tachycardia (PVT) (Fig. 2a). She was febrile during the episodes with a temperature of $39.4{ }^{\circ} \mathrm{C}\left(102.92{ }^{\circ} \mathrm{F}\right)$. Differential diagnosis includes torsade de pointes (TdP) secondary to electrolyte derangement, PVT, PVT secondary to ischemia, feverinduced ventricular tachycardia (VT), TdP secondary to QT prolongation, TdP secondary to underlying channelopathy.

The basic metabolic panel (BMP) drawn at the time of the arrhythmias was significant for sodium $133 \mathrm{mmol} / \mathrm{L}$ (ref. range 136-146), mild hypokalemia with potassium of $3.3 \mathrm{mmol} / \mathrm{L}$ (ref. range $3.6-5.1$ ), bicarbonate $16 \mathrm{mmol} / \mathrm{L}$ (ref. range 23-31), BUN $11 \mathrm{mg} / \mathrm{dL}$ (ref. range 10-20), creatinine $0.5 \mathrm{mg} / \mathrm{dL}$ (ref. range 0.6-1.0), and magnesium $2.8 \mathrm{mg} /$ $\mathrm{dL}$ (ref. range 1.6-2.6). She was given $80 \mathrm{mEq}$ of intravenous potassium after the first episode of torsade. Repeat electrolytes were normal after the second episode.

There were no ECG findings suggestive of ischemia (Fig. $2 \mathrm{~b}, \mathrm{c})$. Serial troponin levels were within normal limits $(<$ $0.03 \mathrm{ng} / \mathrm{mL}$ ). Transthoracic echocardiogram revealed normal left ventricular size, wall thickness, and function. The CT chest/abdomen/pelvis was significant for patchy airspace and ground glass infiltrates in the lungs, most pronounced in the upper lobes, findings most consistent with her ongoing COVID-19.

Following both arrhythmias (atrial fibrillation and TdP), the patient had converted to sinus tachycardia with QTc a

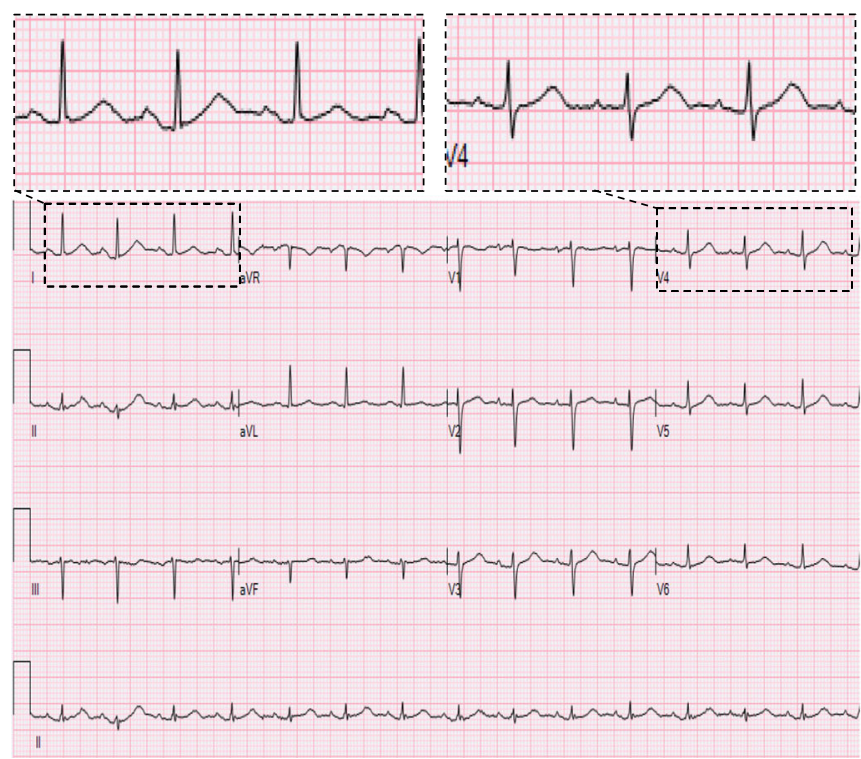

Fig. 1 Representative electrocardiograms of the patient on admission and prior to episodes of torsade de pointes. a Admission electrocardiogram of the patient demonstrating normal sinus rhythm; QTc: $441 \mathrm{~ms}$. b b

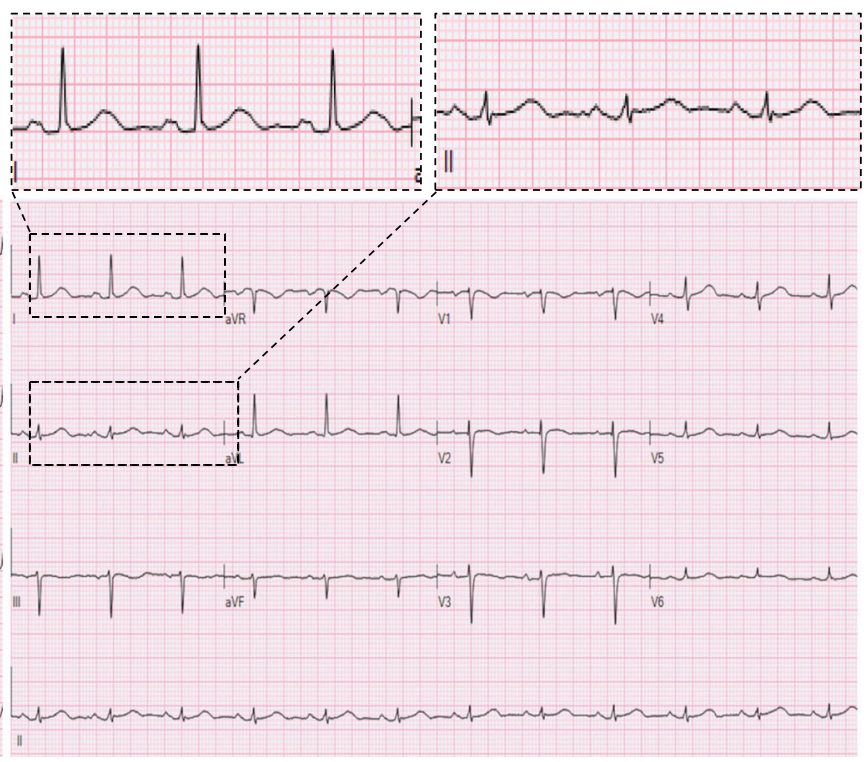

Electrocardiogram of the patient 2 days prior to developing torsade de pointes demonstrating normal sinus rhythm and nonspecific $\mathrm{T}$ wave abnormality in anterior leads. QTc: $467 \mathrm{~ms}$ 
a

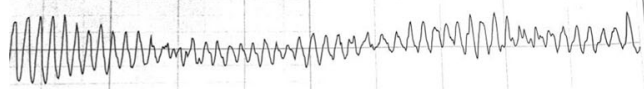

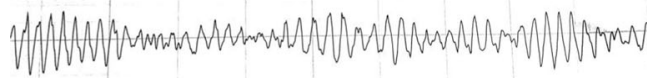

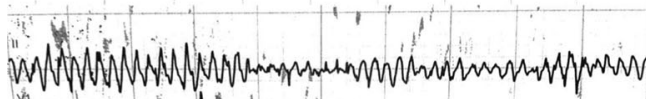

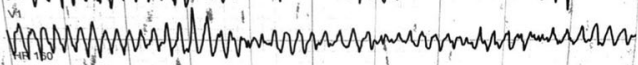

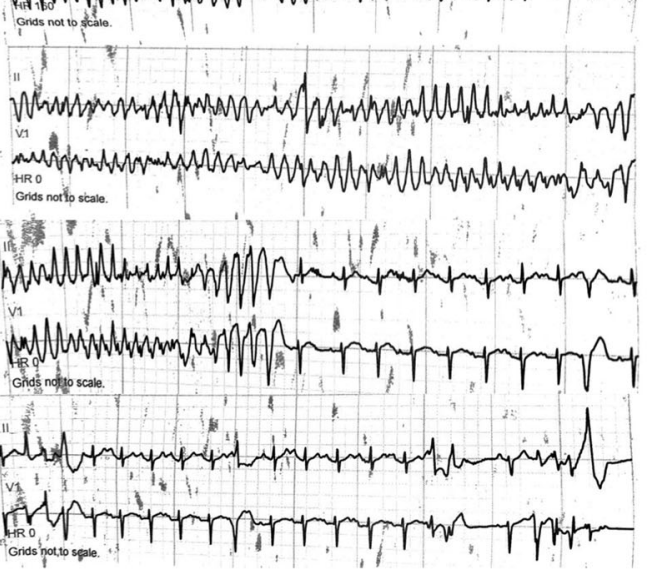

b
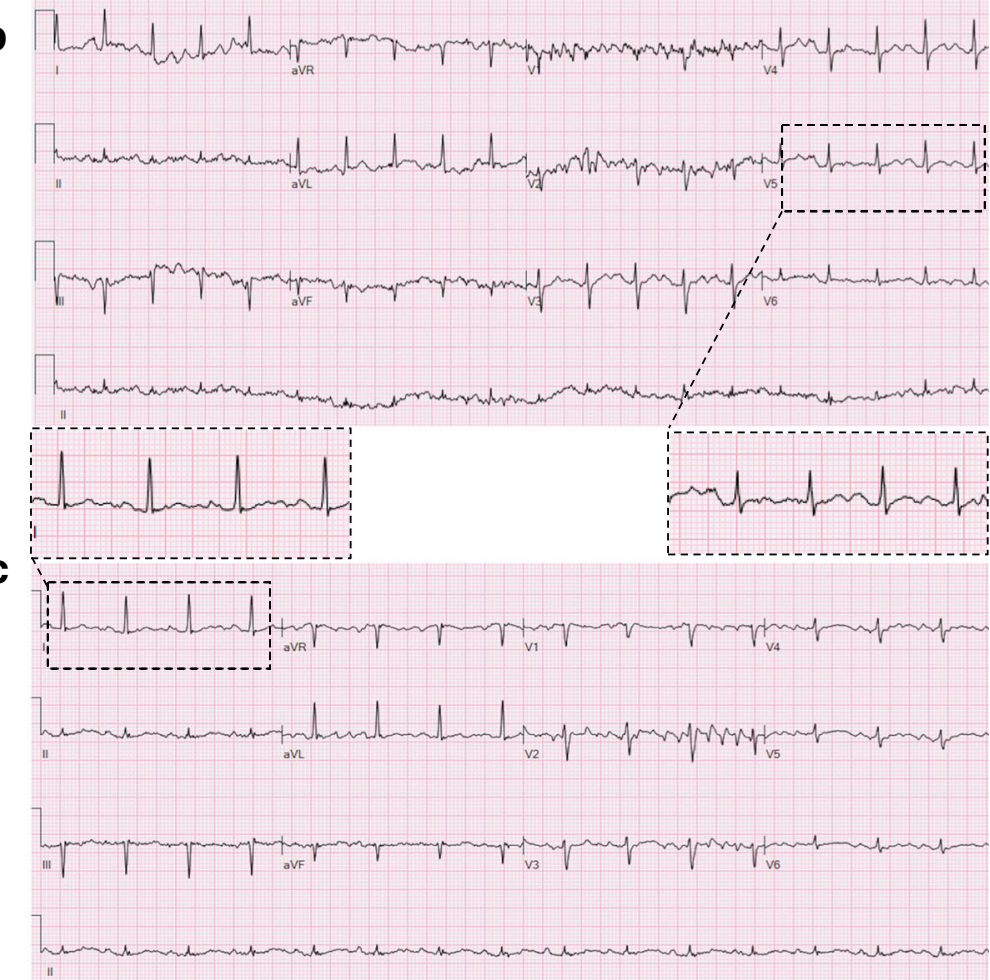

Fig. 2 Representative telemetry strips and electrocardiograms of the patient during and after the episode of torsade de pointes. a Representative telemetry strips of the patient demonstrating first episode (top) and second episode of torsade de pointes (TdP) (bottom 4). b Electrocardiogram of the patient right after TdP was terminated demonstrating sinus tachycardia (123 bpm) and nonspecific ST-T wave abnormality QTc: $380 \mathrm{~ms}$. c Electrocardiogram of the patient a few hours after termination of TdP demonstrating normal sinus rhythm (92 bpm) and nonspecific T wave abnormality QTc: $398 \mathrm{~ms}$ interval of $380 \mathrm{~ms}$ (normal range $<470 \mathrm{~ms}$ ) (Fig. 2b) before medications (including intravenous potassium) or cardioversion could be administered. Since her episodes correlated with high fevers, she was treated aggressively to control the fever using acetaminophen $1000 \mathrm{mg}$ every $6 \mathrm{~h}$. Electrolytes were replenished intravenously with $80 \mathrm{mEq}$ of potassium acetate and $4 \mathrm{~g}$ of magnesium. ECG after electrolyte administration demonstrated normal sinus rhythm with QTe interval of 398 ms (Fig. 2c). After a few hours, her fever subsided. She was transferred to the intensive care unit (ICU). While in the ICU, her potassium and magnesium were closely monitored and corrected to a goal potassium greater than $4.0 \mathrm{mmol} / \mathrm{L}$ and magnesium greater than $2.0 \mathrm{mg} / \mathrm{dL}$. At this point, her temperature continued to be normal with fever control management. After 3 days at the ICU, she was transferred to the telemetry floor without any further episodes of PVT. During hospitalization at our institution, she did not receive hydroxychloroquine or azithromycin.

\section{Discussion}

In patients with COVID-19, hydroxychloroquine or other medications such as azithromycin may be the likely culprit for QT prolongation leading to ventricular arrhythmias. The elimination half-life of hydroxychloroquine and azithromycin is relatively long, more than 40 days and $68 \mathrm{~h}$, respectively [7, 8]. However, QT interval may return to normal once these medications are discontinued. In this case, serial ECGs did not show strong evidence of QTc prolongation.

The first step in the assessment of VT is the identification of treatable and/or reversible causes. Profound hypokalemia and hypomagnesemia can prolong the QT intervals and result in TdP, but in this patient, the levels would not be considered low enough to increase the risk. Moreover, her QTc interval was normal after the episodes of arrhythmia before replenishing potassium making QT prolongation secondary to mild hypokalemia less likely.

To our knowledge, there is no report of spontaneous PVT with TdP morphology in an elderly patient with normal QTc. Also, no cases of fever-induced PVT have been reported in patients with normal QTc.

This is the first case of PVT reported in a COVID-19 patient with normal QTc. It seemed that there was a correlation with fever triggering ventricular tachycardia. We advocated aggressive fever control given the QTc was normal and stable. In general, it is rare to develop PVT with normal QT in the absence of ischemia [4]. Carbapenems, especially imipenem, 
can cause severe hypokalemia in some individuals and can increase the risk of torsade. However, there are no reports of torsade caused by meropenem in the setting of normal QTc [9]. We suspect that this patient might have underlying channelopathy that was unmasked when exposed to stimulants like acute illness secondary to COVID-19 infection. Another possibility would be transient inflammation of the cardiomyocytes causing this arrhythmia. Ischemia is less likely given no ischemic ECG changes, normal echocardiography findings, and normal troponin. Following treatment for presumed superimposed bacterial and fungal infections along with aggressive fever management and supportive therapy for COVID-19 infection, the patient remained in sinus rhythm without further atrial or ventricular arrhythmias.

\section{Conclusions}

Different cardiovascular manifestations have been described in COVID-19 patients including heart failure, arrhythmias, and myocarditis. In the setting of systemic infection, most patients become tachycardic $[2,3]$. They may also develop rhythm abnormalities in the setting of electrolyte imbalance and prolonged QTc due to QT-prolonging medications [10]. Sinus node and atrioventricular node dysfunction has also been reported in stable COVID-19 patients [11]. Even though elderly patients are vulnerable to have serious complications due to negative prognostic factors including hypertension and cardiovascular diseases, cases of successful recovery have been reported [12]. In this case, we present an elderly COVID-19 patient who had normal QTc that developed PVT with TdP appearance. This suggests that COVID-19 patients are prone to different arrhythmias including lifethreatening ventricular arrhythmias with normal left ventricular systolic function and normal QTc. Limitation of this case report is lack of cardiac magnetic resonance imaging to completely rule out myocarditis.

Acknowledgments The authors are grateful to our colleagues, who contributed invaluable clinical information.

\section{Compliance with Ethical Standards}

Conflict of Interest The authors declare that they have no conflict of interest.

Ethical Approval All relevant ethical guidelines have been followed.

Consent Written informed consent was obtained.

\section{References}

1. Spuntarelli V, Luciani M, Bentivegna E, Marini V, Falangone F, Conforti G, et al. COVID-19: is it just a lung disease? A case-based review. SN Compr Clin Med. 2020;28:1-6.

2. Babapoor-Farrokhran S, Gill D, Walker J, Rasekhi RT, Bozorgnia B, Amanullah A. Myocardial injury and COVID-19: possible mechanisms. Life Sci. 2020;117723.

3. Babapoor-Farrokhran S, Tarighati Rasekhi R, Gill D, et al. Arrhythmia in COVID-19. SN Compr Clin Med. 2020;2:1430-5. https://doi.org/10.1007/s42399-020-00454-2.

4. Eisenberg SJ, Scheinman MM, Duller NK, Finkbeiner WE, Griffin JC, Eldar M, et al. Sudden cardiac death and polymorphous ventricular tachycardia in pationts with normal QT intervals and normal systolic cardiac function. Am J Cardiol. 1995;75(10):687-92.

5. Thomas SH, Behr ER. Pharmacological treatment of acquired QT prolongation and torsades de pointes. Br J Clin Pharmacol. 2016;81(3):420-7.

6. Available from: URL: https://crediblemeds.org/

7. Tett SE, Cutler DJ, Day RO, Brown KF. Bioavailability of hydroxychloroquine tablets in healthy volunteers. Br J Clin Pharmacol. 1989 Jun;27(6):771-9.

8. Available from: URL: http://www.drugs.com/pro/zithromax.html

9. Kumar V, Khosla S, Stancu M. Torsade de pointes induced by hypokalemia from imipenem and piperacillin. Case Rep Cardiol. 2017;2017:1-4.

10. Babapoor-Farrokhran S, Alzubi J, Khraisha O, Mainigi SK. Cardiac arrhythmias in the era of COVID-19 pandemic. Trends Cardiovasc Med. 2020. https://doi.org/10.1016/j.tcm.2020.09.001.

11. Babapoor-Farrokhran S, Batnyam U, Wiener PC, Kanjanahattakij N, Khraisha O, Amanullah A, et al. Atrioventricular and sinus node dysfunction in stable COVID-19 patients. SN Compr Clin Med. 2020:1-4. https://doi.org/10.1007/s42399-020-00497-5.

12. Bentivegna E, Luciani M, Spuntarelli V, Speranza ML, Guerritore L, Sentimentale A, Martelletti P. Extremely severe case of COVID19 pneumonia recovered despite bad prognostic indicators: a didactic report.

Publisher's Note Springer Nature remains neutral with regard to jurisdictional claims in published maps and institutional affiliations. 